\title{
La visibilización del sujeto víctima, las instituciones y las luchas políticas por la memoria como categorías de análisis para el estudio de la memoria'
}

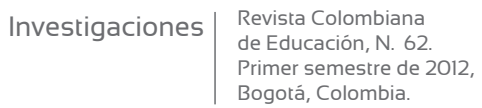

//A visibility of victims, institutions

and political struggles on behalf of memoir as analysis categories

\section{//A visibilização das vítimas, as instituições e as lutas políticas pela memória como categorias de análise para o estudo da memória}

Nathalia Martínez Mora *

Orlando Silva Briceño **
Recibido: 07/11/2011 Evaluado:19/01/2012 - 21/11/2011
*

\begin{abstract}
Magister en Estudios Sociales de la Universidad Pedagógica Nacional y licenciada en Educación Básica con Énfasis en Ciencias Sociales de la Universidad Distrital Francisco José de Caldas. Docente-investigadora de la Universidad Minuto de Dios, vinculada al grupo de investigación Cyberia de la Universidad Distrital Francisco José de Caldas. Correo electrónico: nathaliamartinezm@gmail.com

Candidato a doctor en Educación del Doctorado Interinstitucional en Educación, Universidad Distrital Francisco José de Caldas, magister en Educación con énfasis en Historia de la Educación y la Pedagogía de la Universidad Pedagógica Nacional, licenciado en Ciencias Sociales de la Universidad Distrital Francisco José de Caldas. Docente-investigador del Departamento de Ciencias Sociales de la Universidad Distrital Francisco José de Caldas, vinculado al grupo de investigación Cyberia. Correo electrónico: orlandosilvab@gmail.com
\end{abstract}

\section{Resumen}

El artículo expone resultados de dos procesos de investigación referidos a la memoria del conflicto armado interno colombiano, los cuales se articularon en un mismo proceso analítico. El texto presenta la estrategia o ruta metodológica basada en el enfoque genealógico arqueológico y algunas de las conclusiones derivadas de las investigaciones, organizadas en tres categorías: constitución del sujeto víctima, instituciones de memoria y luchas políticas por la memoria.

\section{Abstract}

This paper offers results of two research processes related and linked analytically to memoir of the Colombian armed conflict. We give a methodological strategy or route based on an archaeological-andgenealogical approach and some research conclusions arranged into three categories: establishment of the victim, memoir institutions, and political struggles on behalf of memoir.

\section{Resumo}

Este artigo expõe resultados de dois processos de investigação sobre a memoria do conflito armado interno colombiano, articulados analiticamente. São apresentadas a estratégia ou rota metodológica baseada no enfoque genealógico-arqueológico e algumas das conclusões derivadas das investigações, organizadas em três categorias: constituição do sujeito-vítima, instituições de memória e lutas políticas pela memória.

\section{Palabras Clave}

Sujeto víctima, instituciones de memoria, luchas políticas por la memoria, enfoque genealógicoarqueológico.

\section{Keywords}

Victim, memoir institutions, political struggles on behalf of memoir, archaeological-andgenealogical approach.

\section{Palavras chave}

Sujeito-vítima, instituições de memória, lutas políticas pela memória, enfoque genealógicoarqueológico. 


\section{Introducción}

El texto presenta parcialmente los resultados de dos investigaciones: "Instituciones y políticas de la memoria sobre el conflicto armado colombiano", desarrollada desde el mes de marzo del 2010 por el Grupo de Investigación Cyberia de la Universidad Distrital Francisco José de Caldas, y la tesis de Maestría en Estudios Sociales titulada "Luchas políticas de la memoria sobre el conflicto armado colombiano", inscrita en la línea de Memoria, identidad y sujetos sociales de la Universidad Pedagógica Nacional. Dichas investigaciones, que fueron articuladas en un solo proceso analítico como parte de la dinámica del grupo Cyberia, permitieron visibilizar la memoria como un campo problémico actual en los escenarios académico, político, social y económico, así como identificar las luchas políticas que sobre esta se generan en el ámbito colombiano en medio del conflicto armado. En este sentido, se observa una creciente producción y discusión sobre la memoria, materializadas en diversas manifestaciones, entre ellas: políticas públicas, proyectos, eventos académicos y comunitarios, desde diversas apuestas y lugares políticos.

Los principales objetivos de las investigaciones fueron determinar cuáles son las iniciativas públicas oficiales y no oficiales, los dispositivos y los discursos que se constituyen en políticas de las memorias sobre el conflicto armado interno colombiano, promovidas por instituciones públicas y organizaciones de la sociedad civil: la Comisión Nacional de Reparación y Reconciliación -CNRR-, la Comisión de Memoria Histórica -CMH-, el Movimiento Nacional de Victimas de Crímenes de Estado -MOVICE-, el Proyecto Colombia Nunca Más -PCNM- y la Asociación de Familiares de Detenidos Desaparecidos -ASFADDES-. Asimismo, identificar y analizar la configuración de las actuales luchas políticas por la memoria sobre el conflicto armado colombiano, sus tendencias y controversias y determinar cuáles son los temas centrales en las propuestas actuales de construcción de memoria sobre el conflicto armado colombiano, con énfasis particular en la masacre de Trujillo, estableciendo sus referentes políticos y los criterios para su elección; razón por la cual se incluyen la Comisión Intercongregacional de Justicia y Paz -CIJP-1 y la Asociación de Familiares Víctimas de Trujillo

Los textos sobre la Masacre de Trujillo revisados, fueron publicados por la Comisión Intercongregacional de Justicia y Paz, pero el archivo de acceso a la documentación se realizó en el Centro de Documentación y en la página Web de la Comisión Intereclesial de Justicia y Paz. 
-AFAVIT-, como instituciones agenciadoras de memoria sobre dicho evento.

Como opción metodológica se recurrió al enfoque genealógico-arqueológico, partiendo de los desarrollos de Michel Foucault, en donde el trabajo se orienta en el análisis de los enunciados sobre la memoria que surgen en las distintas superficies de emergencia (fuentes de información) y su correlación con los enunciados sobre verdad, justicia y reparación (que se hallan en articulación constante con la memoria del conflicto). La selección de este enfoque corresponde a la relación que se puede establecer con el marco teórico escogido y el problema de la investigación. Las estrategias o técnicas que se emplearon fueron la revisión documental, la caracterización institucional y las entrevistas semi-estructuradas. De esta manera, los apartados del artículo se centran en la presentación de la estrategia o ruta metodológica y en algunas de las conclusiones derivadas de las investigaciones, organizadas en las siguientes categorías: constitución del sujeto víctima, instituciones de memoria y luchas políticas por la memoria.

\section{El enfoque genealógico arqueológico: una mirada a la singularidad}

El horizonte metodológico de las investigaciones se basó en el enfoque genealógico-arqueológico, el cual debe comprenderse como una ruta que proporciona orientaciones conceptuales y metodológicas. A partir de este enfoque, se construye el proceso investigativo desde la lógica de la reflexividad y la singularidad, generando un aporte a los campos de investigación en que se enmarca cada proyecto. El trabajo investigativo se establece alrededor de la descripción de acontecimientos discursivos y no discursivos, y no en la interpretación de estos; por tal razón, se opera sobre lo positivamente dicho en el lugar propio de existencia de los enunciados.

Este enfoque podría aproximarse a la investigación cualitativa, aunque es importante establecer algunas de sus diferencias. $\mathrm{Al}$ respecto Félix Recio (1999) señala que la genealogía y arqueología no se sitúan en el nivel del habla, ni de la lengua, contrario al procedimiento cualitativo: estas son formas históricas en donde lo enunciado se ubica en el lugar de aparición para encontrar la singularidad de su emergencia, mientras que el procedimiento cualitativo busca grados de generalización, se basa en estructuras de carácter lógico y pretende la interpretación².

Para Foucault, la arqueología favorece la emergencia de acontecimientos, "pretende definir no los pensamientos, las representaciones,

Según Recio la interpretación puede estar referida a dos clases: una, en la que el discurso sea modificado en cosas distintas, soportada en la representación y en el exceso de significado. Otra, en la que la interpretación se despliega en el círculo interpretativo que no tendría fin, ausente de significado y con preponderancia del significante. 
las imágenes, los temas, las obsesiones que se ocultan o se manifiestan en los discursos, sino esos mismos discursos, esos discursos en tanto prácticas que obedecen a unas reglas [...] no busca 'otro discurso' más escondido" (Foucault, 2005, pp. 233-234), razón por la cual no se establece como un método interpretativo. Por su parte la genealogía, distinta a su significado etimológico que hace referencia al origen, implica para este autor, retomando algunos postulados de Nietzsche:

[...] percibir la singularidad de los sucesos, fuera de toda finalidad monótona; encontrarlos allí donde menos se espera y en aquello que pasa desapercibido por no tener nada de historia -los sentimientos, el amor, la conciencia, los instintos-, captar su retorno, pero en absoluto para trazar la curva lenta de una evolución, sino para reencontrar las diferentes escenas en las que han jugado diferentes papeles; definir incluso el punto de su ausencia, el momento en el que no han tenido lugar (Foucault, 1992, p. 1).

La genealogía, entonces, supone situarse en la emergencia de los acontecimientos discursivos que se dan en los intersticios, dando cuenta del juego o de un determinado campo de fuerzas y luchas sobre ellos. Esta categoría de acontecimiento remite a un conjunto de enunciados que irrumpen de manera singular de acuerdo a unas condiciones específicas de saber y de poder, cuyo análisis parte por reconocer cómo han sido discursivamente constituidos por ciertos enunciados efectivamente producidos que le describen, especifican, precisan, desarrollan y combaten.

Mediante la puesta en marcha de la estrategia analítica y técnicas como la tematización de las fuentes y mapas conceptuales ${ }^{3}$, elaborados para organizar y clasificar la información, la memoria apareció como un acontecimiento construido desde lugares diversos que conformarían una formación discursiva o red de enunciados complejos, caracterizada por la dispersión de sus objetos, conceptos, posiciones de sujeto o tácticas, en condiciones particulares de existencia y cambio. De esta manera, el trabajo analítico consistió en establecer relaciones entre los enunciados o grupos de enunciados que emergieron sobre la problemática investigada: justicia, verdad, reparación, recon-

3 En el desarrollo de este apartado se hará referencia a las estrategias e instrumentos empleados para la recopilación, procesamiento y análisis de la información. 
ciliación, sujeto víctima, así como de las luchas que fijan y hacen posible la memoria del conflicto para el caso colombiano.

En este sentido, la estrategia analítica producida para desarrollar las investigaciones se fue implementando de acuerdo con lo planteado en el enfoque, aunque el proyecto sufrió transformaciones a lo largo del proceso de fundamentación teórica y el tratamiento de la información, que fueron visibilizando opciones de trabajo más propicias según los criterios de viabilidad, pertinencia y aporte social ${ }^{4}$. Dicha estrategia, que se compone de nueve fases, es presentada de forma sintética a continuación.

En primer lugar, se partió de la elaboración de un inventario de las iniciativas de memoria que se estaban generando, especialmente desde el año 2007, pues este periodo fue considerado el umbral de aparición de una gran cantidad de proyectos, programas y eventos, entre otros, sobre la memoria del conflicto armado colombiano. Con la información del inventario se procedió a su organización, agrupación y clasificación, complementándose con lo producido hasta principios del 2011; se identificaron cuatro lu-

4 Para Irene Vasilachis (2006), cada proyecto, sus preguntas y objetivos son susceptibles de transformación según los propósitos que se vayan definiendo, los alcances y el cumplimiento de lo señalado en la planeación de cada estudio. Esta condición, que se aproxima a una de las características de la investigación social, es nominada por la autora el principio de flexibilidad. gares desde los cuales se produce y agencia la memoria sobre el conflicto armado interno colombiano (político, académico, organizativo comunitario y artístico), así como los temas más recurrentes (masacres, víctimas, resistencias, entre otros). Algunos de estos lugares cuentan con mayor difusión, otros con mayor producción de iniciativas, evidenciando que en la actualidad existe una explosión de memoria sobre el conflicto armado.

Posteriormente, se construyó un documento de caracterización de las instituciones oficiales y las organizaciones de víctimas y defensoras de derechos humanos desde las cuales se han adelantado procesos de construcción de memoria del conflicto $^{5}$, junto con un inventario de la información de algunos centros de documentación de estas instituciones, relacionadas con la memoria del conflicto armado y el seguimiento a los procesos judiciales (en el caso de la Comisión Intereclesial de Justicia y Paz), de lo cual se logró establecer, para estas organizaciones, las líneas de trabajo, las

5 Tal y como se mencionó en la introducción, las instituciones que se trabajaron en la investigación fueron: La Comisión Nacional de Reparación y Reconciliación, la Comisión de Memoria Histórica, la Asociación de Familiares Detenidos y Desaparecidos, el Movimiento Nacional de Víctimas de Crímenes de Estado, el Proyecto Colombia Nunca Más, la Comisión Intereclesial de Justicia y Paz, la Comisión Intercongregacional de Justicia y Paz y la Asociación de Familiares Víctimas de Trujillo; cabe anotar que el proceso analítico en cada una se particularizó de acuerdo a su producción escrita, visual, sonora, artística, entre otras. 
estrategias utilizadas, los planes de acción y algunas maneras de operar frente a los procesos de reconstrucción de memoria; esta información fue complementada con las entrevistas realizadas a algunos miembros pertenecientes a ellas. De la caracterización, acompañada de la relación con el trabajo conceptual, emergió la categoría de instituciones de memoria, vista como un aporte teórico para analizar las condiciones propias de producción y agenciamiento de memoria sobre el conflicto armado en el país.

Seguido a ello, se delimitó el corpus analítico necesario para la investigación. A través de este se determinaron, desde lo positivamente enunciado, las discusiones, diferencias, debates, similitudes en los discursos y en los referentes políticos. Este proceso facilitó la visibilización de relaciones de unos enunciados con otros, entre grupos de enunciados de una misma formación discursiva o entre enunciados de campos distintos (Foucault, 2005); por ejemplo, la relación entre memoria social e histórica y memoria judicial, sujeto víctima y sujeto de derechos o emprendedores de memoria y víctimas. De los documentos seleccionados (entrevistas, textos, instalaciones, videos) en el corpus, se emprendió un proceso de descripción de los discursos que circulaban sobre la memoria del conflicto, y en algunos casos con énfasis particular en la masacre de Trujillo, que permitieron la aparición de categorías relacionadas con la memoria: verdad, justicia, reparación, mediante la elaboración de fichas temáticas, bibliográficas y analíticas.

Esta fase se concretó en varios documentos que fueron reelaborados desde las categorías teóricas y las conexiones con las categorías que emergían en los documentos. Conforme al enfoque se definió un nivel de análisis que consistía en contrastar las categorías teóricas con los enunciados y discursos que aparecían en las superficies de emergencia utilizadas. A partir de esto, se realizaron mapas relacionales tomando como base el esquema de instituciones, sujetos y discursos, dándose consecución al trabajo analítico de los enunciados sobre la memoria del conflicto armado interno colombiano.

En la última fase, se desarrolló el proceso de escritura y de organización del informe, que contó con la socialización de los avances y resultados de las investigaciones en eventos nacio- 
nales e internacionales ${ }^{6}$. También se hicieron documentos que contenían reflexiones sobre el proceso investigativo y la configuración de la estrategia metodológica apropiada para cada proyecto. Estas socializaciones hacen parte de la estrategia comunicativa de los resultados de la investigación.

Finalmente, como se verá en los siguientes apartados, la emergencia de la memoria del conflicto, que se define por una irrupción conflictiva y nunca acabada, está sustentada por un saber que va siendo constituido por sujetos de memoria (en este documento se aborda el sujeto víctima), fijada por las instituciones de memoria y configurada por un campo de fuerzas que la atraviesan y delimitan, en lo que se ha denominado luchas políticas por la memoria (Jelin, 2002). Estos tres procesos identificados (sujeto víctima, instituciones de memoria y luchas políticas por la memoria) se constituyen en categorías analíticas imprescindibles para abordar los estudios sobre la memoria.

6 El año 2010 se participó en el XV Congreso Colombiano de Historia. Asociación Colombiana de Historiadores. Bogotá 26-30 de julio de 2010, con la ponencia "Políticas de la memoria: prácticas institucionales y construcción de sentidos sobre el pasado reciente del conflicto interno armado colombiano" y en el $7^{\circ}$ Foro Latinoamericano "Memoria e identidad" Caminos de encuentro entre pueblos y culturas. SIGNO Centro Interdisciplinario. Montevideo (Uruguay) 28 al 31 de octubre de 2010, con la ponencia: "Políticas de la memoria sobre el conflicto armado colombiano: la construcción de otros relatos".

\section{Constitución del sujeto víctima y la reparación como efecto de la dimensión política de la víctima}

En la consolidación de los procesos de instauración de prácticas de la memoria relacionadas con el conflicto armado interno en la última década en Colombia, se han configurado un conjunto de estrategias, instituciones, campos discursivos y luchas políticas que promovieron la emergencia de nuevos agentes discursivos, que apoyados en tecnologías de subjetivación y teniendo como uno de sus ejes constitutivos diferentes prácticas de memoria, produjeron distintos "sujetos de memoria". Uno de dichos tipos de subjetividad es el que se identifica a partir de la emergencia del sujeto víctima, el cual ha adquirido gran preponderancia en los debates públicos, producciones académicas, movilizaciones sociales, reconocimientos normativos y legislativos.

En las investigaciones se evidencia que este acontecimiento (emergencia del sujeto víctima), recurrente en otros países en donde ha sido significativo el asunto de la memoria colectiva vinculada a procesos de posconflicto o de transición a la democracia, o bien a procesos traumáticos para la sociedad como el caso de atentados o hechos violentos, conlleva diversidad de tensiones y problemas que han derivado en dis- 
tintas posiciones en relación a la condición de dicho sujeto en la esfera pública. Si bien se reconoce que vivimos en el tiempo de las víctimas (Eliacheff y Soulez, 2009), dicho reconocimiento es ambiguo. En un sentido, se sigue utilizando desde una perspectiva de la compasión: "Existe, pues, una relación entre compasión, democracia y víctimas [...] La compasión nacida de los principios democráticos parece favorecer la democracia. Los derechos reconocidos de las víctimas tienen como objetivo caminar en el sentido de la igualdad" (Eliacheff y Soulez, 2009, p. 15).

Por otra parte, el reconocimiento social de las víctimas tiene fuertes componentes políticos y jurídicos: "Las víctimas cada vez están más presentes en nuestros discursos. Ya no es un asunto de piedad [...] sino que forman parte de nuestro paisaje, particularmente del jurídico" (Mate, 2003, p. 100). También son reconocidas las discusiones en torno a las posibilidades de manipulación e instrumentalización de quienes se autoreconocen o son reconocidas como víctimas.

Para el caso colombiano, la preponderancia de las víctimas del conflicto armado es en gran parte producto del papel que han jugado las asociaciones que tienen como eje de reivindicación dicha condición, tal es el caso del MOVICE, AFAVIT, ASFADDES, CIJP, PCNM. Por ejemplo, para el MOVICE, movimiento conformado por organizaciones y sobrevivientes de crímenes de Estado en Colombia, su objetivo fundamental consiste en "reivindicar a las víctimas de crímenes cometidos por el Estado sea por su acción u omisión, en acciones perpetradas por miembros de la fuerza pública o fuerzas paraestatales o paramilitares que actuaron bajo el auspicio, respaldo y connivencia del Estado colombiano en diversas zonas del país" (MOVICE, 2010). Desde allí, este movimiento, fundamenta sus acciones en la exigencia del "restablecimiento de los derechos a la verdad, justicia, reparación integral, garantía de no repetición y a la memoria de centenares de miles de hombres y mujeres que le apostaron a construir una Colombia en paz y con justicia social." (MOVICE, 2010)

Las asociaciones de víctimas a nivel internacional aparecieron en 1983 (Eliacheff y Soulez, 2009, p. 47) y han jugado un papel primordial en la visibilidad y centralidad de ellas en la sociedad:

[...] la función de las asociaciones va más allá de los fines descritos en sus estatutos. Su fuerza estriba en haber conseguido que la víctima pasara de ser ignorada 
a ser reconocida. Sabemos que la ausencia de reconocimiento puede provocar heridas tan intensas como el propio traumatismo. Sufrir no basta para conseguir la reparación. El testimonio del reconocimiento no llega si no se actúa. Las asociaciones se encargaron de conseguir el reconocimiento de las víctimas allí donde reinaba la ocultación, la discriminación y la estigmatización (Eliacheff y Soulez, 2009, p. 48).

El componente político que resulta del reconocimiento, visibilidad y protagonismo de las víctimas en las actuales condiciones de las justicias restaurativas, pasa por el papel que han jugado las prácticas memorísticas, impulsadas desde las iniciativas de memoria de las asociaciones, o de las organizaciones oficiales que se constituyen en instituciones de memoria.

Para el caso de AFAVIT, asociación conformada por familiares y víctimas de la masacre de Trujillo ${ }^{7}$, el objetivo central de los trabajos de memoria que ellos impulsan consis-

7 La masacre de Trujillo ha sido tipificada por la Comisión Interamericana de Derechos Humanos como el conjunto de homicidios, desapariciones forzadas, torturas y desplazamientos sucedidos en los municipios de Trujillo, Bolívar y Riofrio (Valle del Cauca) entre los años 1988 a 1990, aunque las organizaciones de víctimas afirman que la masacre sucedió entre 1988 a 1999. te en reconstruir el tejido personal y social, lograr el respeto a la dignidad de las personas y familias afectadas y buscar que se cumplan efectivamente los derechos a la verdad, la justicia y la reparación. Desde este objetivo asumen primordial la elaboración de memoria desde las víctimas, pues sostienen que "las convierte en sujetos históricos, agentes activos de su propio cambio, siendo la memoria la fuente, la conciencia de la humanidad. Quienes luchan por sus derechos, luchan por la Verdad, la Justicia y una Reparación Integral"8, lo que comporta un carácter psicológico, ético, religioso y político.

En los debates actuales sobre las víctimas se empieza a reconocer como esencial la significación política de las mismas: "[...] la víctima tiene, en el hecho mismo de ser víctima, una significación política" (Mate, 2008, p. 7) y a su vez, al otorgársele esa significación política, los procesos reparativos de la sociedad dirigidos a las víctimas son ineludibles. En este sentido, el principio de reparación es también efecto del reconocimiento político de las víctimas, y no solo por el principio de resarcimiento que ha instaurado los modelos de justicia restaurativa, pues en la esfera política el carácter

8 Hna. Maritze Trigos, acompañante en Trujillo. En: Encuentro- Diálogos con las víctimas en clave Psicosocial., organizado por el Ministerio de Protección Social, la Conferencia Episcopal de Colombia y el Secretariado Nacional de Pastoral Social Cáritas Colombiana. Cali-Valle, noviembre 22 de 2010.

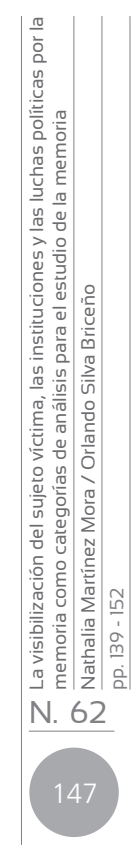


simbólico de la reparación pasa por la dignificación de las víctimas, que pretende superar las prácticas y políticas compasivas frente a ellas.

\section{Instituciones de memoria}

La condición estratégica de la memoria, en el panorama de lo que Foucault plantea como una ontología del presente centrada en la pregunta por aquello que constituye nuestra actualidad, ha producido el surgimiento de lo que hemos denominado, a partir de las investigaciones, instituciones de memoria (Martínez y Silva, 2012), las cuales irrumpen en el espacio público y generan multiplicidad de iniciativas, promoviendo una reactualización del pasado en el presente de acontecimientos conflictivos.

Las instituciones de memoria hacen parte de los dispositivos sociales que operan como agentes productores y distribuidores de políticas de la memoria del conflicto armado, que mediante variadas estrategias, construyen multiplicidad de sentidos sobre el pasado reciente colombiano e instituyen un conjunto de prácticas discursivas sobre los significados de lo acontecido:

Estas instituciones, que no se limitan a una existencia material, se hallan en relación constante con los discursos, otras instituciones y los sujetos de saber, quienes ponen en juego los enunciados y las prácticas de saber sobre el conflicto armado interno colombiano y despliegan un orden del discurso [...] de manera que los discursos sobre el conflicto armado que son producidos por las instituciones de memoria se hallan enmarcados bajo sistemas de exclusión, estableciendo regímenes de verdad acerca de este fenómeno, es decir, determinando el saber que puede circular, fijando sus límites, las condiciones de su utilización y precisando el lugar de enunciación de los sujetos que tienen validez para pronunciar ese saber. (Martínez y Silva, 2012).

En las investigaciones se pudo determinar que el conjunto de organizaciones de la sociedad civil, asociaciones de víctimas e instancias oficiales trabajadas, son instituciones de memoria que vienen produciendo y agenciando proyectos, eventos, instalaciones, reivindicaciones y demás, sobre el conflicto armado in- 
terno en el país ${ }^{9}$. Esta condición permite a su vez la instauración de órdenes discursivos, la fijación de límites a las prácticas discursivas y el establecimiento de un campo de fuerzas o luchas políticas que constituyen la memoria del conflicto para el caso colombiano.

\section{Luchas políticas por la memoria}

El campo de la memoria consolidado en Colombia en las últimas décadas ha estado atravesado por continuas discusiones y confrontaciones teóricas, políticas, éticas y jurídicas, que configuran su singularidad y lo complejizan. Los distintos debates y las implicaciones sociales que ellos conllevan, sobre todo en relación a los sentidos y significados del conflicto interno armado y las responsabilidades del Estado, la sociedad civil y los distintos actores sociales, hacen de este campo un escenario de lucha: "Las luchas políticas inician con el acontecimiento violento o conflictivo, cuyo eje de disputa se centra en la interpretación que se produzca sobre este, y en su representación, comportando cuestiones

9 Una caracterización de las iniciativas y procesos de memoria que componen el campo de producción de las instituciones de memoria es presentada de forma detallada en el texto titulado "Instituciones de memoria sobre el conflicto armado colombiano en la producción de iniciativas y constitución discursiva de sujetos". En: Castillejo, A. (Edit.) (2012). Violencia, Memoria y Sociedad: Debates y Agendas en las Colombia Actual. Bogotá: Universidad de los Andes. (Capítulo de libro en prensa) acerca del cómo debe ser transmitido y quién decidirá qué y cómo se hará" (Martínez y Silva, 2012).

En el ámbito internacional, esta categoría ha sido utilizada para analizar las tensiones, polémicas y combates que se dan por los sentidos de un pasado -usualmente traumático-. Elizabeth Jelin concibe la conformación de estas luchas en un contexto caracterizado por las aperturas políticas ${ }^{10}$, que permite la manifestación y revelación de diferentes actores, reivindicaciones y relatos sobre este pasado, como sucedió en los periodos de transición a un régimen constitucional, post-dictatoriales o de una postguerra civil, desde la segunda mitad del siglo XX en Alemania, España, Brasil o los países del Cono Sur. Para el caso colombiano, se expresa un rasgo singular de cara a estas luchas políticas, pues si bien el país se inscribe en el marco de una democracia establecida jurídicamente, esto no desconoce la existencia de una multiplicidad de luchas políticas por la memoria sobre un conflicto armado interno caracterizado por constantes violaciones a los derechos humanos a través de acciones sistemáticas y masivas de eliminación y fijación del miedo en las poblaciones.

De esta manera, en los procesos investigativos se determinó que las producciones y elaboraciones de

10 Este contexto de aperturas políticas generalmente promueve un escenario político de cambio institucional en el Estado y en la relación sociedad- Estado, apuntando su configuración hacia la democracia. 
memoria muestran la presencia de estas luchas que se expresan en distintos horizontes. Uno de ellos se establece alrededor del acontecimiento conflictivo en sí mismo, es decir, su interpretación y la manera como es presentado. También en relación con las acciones judiciales promovidas, los reconocimientos simbólicos y las fechas (de las masacres o instigamientos sobre la sociedad civil), los aniversarios y las conmemoraciones. Para Jelin, de la interpretación de los acontecimientos conflictivos se formula la cuestión de cómo encarar ese pasado que se constituye en eje de disputas de estrategias políticas distintas.

Otro de los horizontes en donde se expresan las luchas políticas es la nominación de los acontecimientos, la periodización, el número de víctimas determinado, la nominación de las personas y poblaciones afectadas (sujetos-víctimas, sujetos de derecho), la presencia de distintos actores políticos que buscan un reconocimiento social y la legitimación de su versión o narrativa del pasado, el contenido dominante de los acontecimientos, la búsqueda de reivindicaciones y reparaciones alrededor del papel de la memoria en estos procesos. En suma, se podría decir que estas luchas van definiendo parte de las estrategias e iniciativas de memoria que se proponen para generar la aceptación en el ámbito público del sentido o significado que se agencia (Martínez y Silva, 2012), así como van configurando las políticas de la memoria sobre este conflicto, en relación con el nivel de negociación que se logre en el terreno de la memoria colectiva.

\section{Conclusiones}

El campo de la memoria al ser uno de los ámbitos de mayor debate y actualidad en los escenarios académicos, políticos, sociales y económicos del país, permite decantar a partir de la creciente y prolífica producción de iniciativas de memoria que se han implementado en los últimos años, una serie de categorías que van constituyendo el horizonte analítico de dicho campo.

En los procesos de investigación, que dieron origen a este artículo, se determinaron como categorías de análisis imprescindibles para los estudios de memoria sobre el conflicto interno armado, las siguientes: constitución del sujeto víctima, instituciones de memoria y luchas políticas por la memoria. 
La primera categoría, visibiliza los hallazgos referidos a la constitución de los sujetos del conflicto armado, en el que es central el de sujeto-víctima, que emerge a partir de los procesos impulsados por las instituciones de memoria en el marco de diversos procesos de subjetivación y por efecto de la disputa jurídica y el reconocimiento social y político de las personas que luchan por dicho reconocimiento ante el Estado colombiano.

La segunda, surge de la evidencia de cómo las organizaciones sociales e instancias oficiales que agencian procesos e iniciativas de memoria sobre el conflicto armado, se constituyen en instituciones de memoria, en tanto que configuran órdenes discursivos con pretensión de regímenes de verdad, determinando el saber que puede circular, como forma de memoria social, fijando sus límites, las condiciones de su utilización y su lugar en las luchas políticas y atribuyendo el lugar de enunciación de los sujetos que tienen validez para pronunciar ese saber.

La última categoría, permite comprender la complejidad y las dinámicas de tensión que disponen el campo de la memoria como un escenario de combate; las luchas políticas por la memoria, expresan los nodos de tensión sociales y políticos, que se manifiestan en la continua resignificación de los hechos conflictivos, las fechas y conmemo- raciones, los lugares de memoria, en fin el conjunto de significados sobre el pasado reciente del conflicto armado interno colombiano.

\section{Referencias}

Eliaacheff, C. y Soulez, D. (2009). El tiempo de las víctimas. Madrid: Akal.

Foucault, M. (1992). Microfísica del Poder. Madrid: Ediciones de La Piqueta.

Foucault, M. (2005). Arqueología del saber. México: Siglo XXI.

Jelin, E. (2002). Los trabajos de la memoria. España: Siglo XXI.

Martínez, N. y Silva, O. (2012) Instituciones de memoria sobre el conflicto armado colombiano en la producción de iniciativas y constitución discursiva de sujetos. En: Castillejo, A. (Edit.) (2012). Violencia, memoria y sociedad: Debates y agendas en la Colombia actual. Bogotá: Universidad de los Andes (Capítulo de libro en prensa).

MOVICE. (2008). Nuestra identidad. Archivo en versión pdf. Pág. 1. Disponible en http: / /www.movimientode victimas. org. Consultado el 22 de junio de 2010.

Recio, F. (1999). Análisis de la realidad social. En: Métodos y técnicas cualitativos de investigación en ciencias sociales. El enfoque arqueológico y genealógico. Editorial Síntesis, tercera edición. 
Reyes, M. (2003). En torno a una justicia anamnética. En: Mardones, J M. y Reyes, M. La ética ante las víctimas. Barcelona: Anthropos.

Reyes, M. (2008). Justicia de las víctimas: terrorismo, memoria, reconciliación. Barcelona: Anthropos.

Vasilachis, I. (2006). Estrategias de investigación cualitativa. Barcelona: Gedisa. 